Volume 132, Number 11, Pages 3309-3318

S 0002-9939(04)07479-9

Article electronically published on May 12, 2004

\title{
HYPERBOLIC DERIVATIVES AND GENERALIZED SCHWARZ-PICK ESTIMATES
}

\author{
PRATIBHA GHATAGE AND DECHAO ZHENG \\ (Communicated by Joseph A. Ball)
}

\begin{abstract}
In this paper we use the beautiful formula of Faa di Bruno for the $n$th derivative of composition of two functions to obtain the generalized Schwarz-Pick estimates. By means of those estimates we show that the hyperbolic derivative of an analytic self-map of the unit disk is Lipschitz with respect to the pseudohyperbolic metric.
\end{abstract}

\section{INTRODUCTION}

For each $z \in D$, let $\varphi_{z}$ denote the Möbius transformation of $D$,

$$
\varphi_{z}=\frac{z-w}{1-\bar{z} w}
$$

for $w \in D$. The pseudo-hyperbolic distance on $D$ is defined by

$$
\rho(z, w)=\left|\varphi_{z}(w)\right|, \quad z, w \in D .
$$

The pseudohyperbolic distance is Möbius invariant, that is,

$$
\rho(g z, g w)=\rho(z, w),
$$

for all $g \in \operatorname{Aut}(D)$, the Möbius group of $D$, and all $z, w \in D$. It has the following useful property:

$$
1-\rho(z, w)^{2}=\frac{\left(1-|z|^{2}\right)\left(1-|w|^{2}\right)}{|1-\bar{z} w|^{2}} .
$$

The Bergman metric on $D$ is the hyperbolic metric whose element of length is

$$
d s=\frac{|d z|}{1-|z|^{2}} .
$$

In this metric every rectifiable arc $\gamma$ has a length

$$
\int_{\gamma} \frac{|d z|}{1-|z|^{2}}
$$

It is easy to show that the induced distance on $D$ is given by

$$
\beta(z, w)=\frac{1}{2} \log \frac{1+\rho(z, w)}{1-\rho(z, w)},
$$

for $z, w \in D$.

Received by the editors July 9, 2003 and, in revised form, August 12, 2003.

2000 Mathematics Subject Classification. Primary 30C80.

The second author was supported in part by the National Science Foundation. 
Let $\varphi$ be an analytic self-map of the unit disk. By the classical Schwarz-Pick Lemma [2] [5], $\varphi$ decreases the hyperbolic distance between two points and the hyperbolic length of an arc. The explicit inequality is

$$
\left|\frac{\varphi\left(z_{1}\right)-\varphi\left(z_{2}\right)}{1-\overline{\varphi\left(z_{1}\right)} \varphi\left(z_{2}\right)}\right| \leq\left|\frac{z_{1}-z_{2}}{1-\overline{z_{1}} z_{2}}\right|
$$

for any $z_{1}, z_{2}$ in $D$. In particular,

$$
\frac{\left|\varphi^{\prime}(z)\right|}{1-|\varphi(z)|^{2}} \leq \frac{1}{1-|z|^{2}}
$$

for $z$ in $D$. Let

$$
\tau_{\varphi}(z)=\frac{\left(1-|z|^{2}\right) \varphi^{\prime}(z)}{1-|\varphi(z)|^{2}}
$$

Then

$$
\left|\tau_{\varphi}(z)\right| \leq 1,
$$

for all $z \in D$. Nontrivial equality $\left|\tau_{\varphi}(z)\right|=1$ holds for some $z \in D$ only when $\varphi$ is a fractional linear transformation $e^{i \theta} \varphi_{a}(z)$. For each $z \in D$, the hyperbolic derivative of $\varphi$ at $z$ is defined by

$$
\lim _{\beta(z, w) \rightarrow 0} \frac{\beta(\varphi(z), \varphi(w))}{\beta(z, w)} .
$$

In Section 3 we will show that the hyperbolic derivative of $\varphi$ equals $\left|\tau_{\varphi}(z)\right|$ and that $\tau_{\varphi}(z)$ is Lipschitz with respect to the pseudohyperbolic metric. Hyperbolic derivatives have been used in studying composition operators on the Bloch space [7], [9] and [10].

Recently, MacCluer, Stroethoff, and Zhao [8] used the formula of Faa di Bruno and the theory of the weighted composition operators [1] to obtain the generalized Schwarz-Pick estimates:

$$
\sup _{z \in D} \frac{\left(1-|z|^{2}\right)^{n}\left|\varphi^{(n)}(z)\right|}{\left(1-|\varphi(z)|^{2}\right)}<\infty
$$

for each analytic self-map $\varphi$ of the unit disk. We obtain the following generalized Schwarz-Pick estimates: for each $0<r<1$ and each positive integer $n$, there is a positive constant $M_{n, r}$ such that for each analytic self-map $\varphi$ of the unit disk:

$$
\frac{\left(1-|z|^{2}\right)^{n}\left|\varphi^{(n)}(z)\right|}{1-|\varphi(z)|^{2}} \leq M_{n, r} \max _{\rho(w, z)<r} \frac{\left(1-|w|^{2}\right)\left|\varphi^{\prime}(w)\right|}{1-|\varphi(w)|^{2}},
$$

for $z$ in $D$. Clearly, Combining (1.2) with (1.4) gives (1.3). Moreover, (1.4) directly leads to the result [8] that if $\varphi$ is in the little Bloch class, then

$$
\lim _{|z| \rightarrow 1} \frac{\left(1-|z|^{2}\right)^{n}\left|\varphi^{(n)}(z)\right|}{1-|\varphi(z)|^{2}}=0
$$

for each $n$. The main tool is the beautiful formula of Faa di Bruno [13] for the $n$th derivative of the composition of two functions.

Based on the generalized Schwarz-Pick estimates we will show in Section 3 that $\tau_{\varphi, n}(z)=\frac{\left(1-|z|^{2}\right)^{n}\left|\varphi^{(n)}(z)\right|}{\left(1-|\varphi(z)|^{2}\right)}$ is Lipschitz with respect to the pseudohyperbolic metric. Thus $\tau_{\varphi, n}(z)$ admits a continuous extension to the set of nontrivial Gleason parts of the maximal ideal space of $H^{\infty}$. 


\section{Generalized Schwarz-Pick estimates}

In this section, we will present a proof of the generalized Schwarz-Pick estimates. The generalized Schwarz-Pick estimates will be used in the proof of Theorem [6 The main tool is the beautiful formula of Faa di Bruno, which deals with the $n$th derivative of composition of an analytic function $f$ on the unit disk with a self-map $\varphi$ of the the unit disk 13 .

Theorem 1 (The Formula of Faa di Bruno). If $\varphi$ is an analytic function from the unit disk to the unit disk and if $f$ is an analytic function on the unit disk, then

$$
(f \circ \varphi)^{(n)}(z)=\sum \frac{n !}{k_{1} ! k_{2} ! \cdots k_{n} !} f^{(k)}(\varphi(z)) \prod_{j=1}^{n}\left(\frac{\varphi^{(j)}(z)}{j !}\right)^{k_{j}}
$$

where $k=k_{1}+\cdots+k_{n}$ and the sum is over all $k_{1}, \ldots, k_{n}$ for which $k_{1}+2 k_{2}+\cdots+$ $n k_{n}=n$.

The following result is well known [12]. We include a proof to motivate our Theorem 2

Proposition 1. If $\varphi$ is a univalent analytic self-map of $D$, then

$$
\left(1-|z|^{2}\right)\left|\varphi^{\prime \prime}(z)\right| \leq 10\left|\varphi^{\prime}(z)\right|
$$

for all $z \in D$.

Proof. For a fixed $z$ in $D$, let $h$ be the Koebe transform of $\varphi$,

$$
h(w)=\frac{\varphi\left(\frac{w+z}{1+z w}\right)-\varphi(z)}{\left(1-|z|^{2}\right) \varphi^{\prime}(z)} .
$$

Then $h(0)=0$ and $h^{\prime}(0)=1$. It follows from Bieberbach's theorem ([12], page 8) that

$$
\left|h^{\prime \prime}(0)\right| \leq 4
$$

On the other hand, an easy computation gives

$$
h^{\prime \prime}(0)=\frac{1}{2}\left(1-|z|^{2}\right) \frac{\varphi^{\prime \prime}(z)}{\varphi^{\prime}(z)}-\bar{z}
$$

Hence

$$
\left|\frac{1}{2}\left(1-|z|^{2}\right) \frac{\varphi^{\prime \prime}(z)}{\varphi^{\prime}(z)}-\bar{z}\right| \leq 4
$$

Since $|z| \leq 1$, we conclude that

$$
\left|\left(1-|z|^{2}\right)\right| \varphi^{\prime \prime}(z)|\leq 10| \varphi^{\prime}(z) \mid .
$$

This completes the proof.

As a consequence of the proposition, we have

$$
\frac{\left(1-|z|^{2}\right)^{2}\left|\varphi^{\prime \prime}(z)\right|}{1-|\varphi(z)|^{2}} \leq \frac{10\left(1-|z|^{2}\right)\left|\varphi^{\prime}(z)\right|}{1-|\varphi(z)|^{2}}
$$

for all $z \in D$ if $\varphi$ is a univalent self-map of the unit disk. 
Example. Let $b$ be an interpolating Blaschke product with zeros $\left\{z_{n}\right\}$ in the unit disk and $\varphi=b^{2}$. Clearly, $\varphi^{\prime}\left(z_{n}\right)=0$ and $\varphi^{\prime \prime}\left(z_{n}\right)=2\left[b^{\prime}\left(z_{n}\right)\right]^{2}$. Let $\delta=$ $\inf _{z_{n}}\left(1-\left|z_{n}\right|^{2}\right)\left|b^{\prime}\left(z_{n}\right)\right|$. Thus

$$
\frac{\left(1-\left|z_{n}\right|^{2}\right)\left|\varphi^{\prime \prime}\left(z_{n}\right)\right|}{1-\left|\varphi\left(z_{n}\right)\right|^{2}}=2\left(1-\left|z_{n}\right|^{2}\right)\left[b^{\prime}\left(z_{n}\right)\right]^{2} \geq 2 \delta\left|b^{\prime}\left(z_{n}\right)\right| \geq \frac{2 \delta^{2}}{1-\left|z_{n}\right|^{2}} .
$$

So the inequality (2.1) does not hold for some analytic self-maps of the unit disk. But by means of the formula of Faa di Bruno we still have the generalized SchwarzPick estimates:

Theorem 2. For each positive integer $n$ and each number $0<r<1$, there is a positive constant $M_{n, r}$ such that for each analytic self-map $\varphi$ of the unit disk,

$$
\frac{\left(1-|z|^{2}\right)^{n}\left|\varphi^{(n)}(z)\right|}{1-|\varphi(z)|^{2}} \leq M_{n, r} \max _{\rho(w, z)<r} \frac{\left(1-|w|^{2}\right)\left|\varphi^{\prime}(w)\right|}{1-|\varphi(w)|^{2}}
$$

for $z$ in $D$.

As we mentioned in the introduction, by the Schwarz-Pick estimates (1.2), we have

$$
\frac{\left(1-|w|^{2}\right)\left|\varphi^{\prime}(w)\right|}{1-|\varphi(w)|^{2}} \leq 1
$$

Thus Theorem 2 implies the following generalized Schwarz-Pick Estimates [8].

Theorem 3. For each $n$, there is a positive constant $M_{n}$ such that for each analytic self-map $\varphi$ of the unit disk,

$$
\frac{\left(1-|z|^{2}\right)^{n}\left|\varphi^{(n)}(z)\right|}{1-|\varphi(z)|^{2}} \leq M_{n}
$$

for $z$ in $D$.

If $\varphi$ is in the little Bloch class, i.e.,

$$
\frac{\left(1-|z|^{2}\right)\left|\varphi^{\prime}(z)\right|}{1-|\varphi(z)|^{2}} \rightarrow 0
$$

as $|z| \rightarrow 1$, then noting that for the given $0<s<1$, for every $w \in D$ with $\rho(w, z)<s,|w| \rightarrow 1$ as $|z| \rightarrow 1$, Theorem 2 gives the following result in [8].

Theorem 4. Let $\varphi$ be an analytic self-map of the unit disk. If

$$
\lim _{|z| \rightarrow 1^{-}} \frac{\left(1-|z|^{2}\right)\left|\varphi^{\prime}(z)\right|}{1-|\varphi(z)|^{2}}=0
$$

then for each $n$,

$$
\lim _{|z| \rightarrow 1^{-}} \frac{\left(1-|z|^{2}\right)^{n}\left|\varphi^{(n)}(z)\right|}{1-|\varphi(z)|^{2}}=0 .
$$

Proof of Theorem 圆 For a fixed $z$ in $D$, let $g=\varphi \circ \varphi_{z}$. Clearly, $g(0)=\varphi(z)$. By the formula of Faa di Bruno, we have

$$
g^{(n)}(w)=\sum \frac{n !}{k_{1} ! k_{2} ! \cdots k_{n} !} \varphi^{(k)}\left(\varphi_{z}(w)\right) \prod_{j=1}^{n}\left(\frac{\varphi_{z}^{(j)}(w)}{j !}\right)^{k_{j}}
$$

where $k=k_{1}+\cdots+k_{n}$ and the sum is over all $k_{1}, \ldots, k_{n}$ for which $k_{1}+2 k_{2}+\cdots+$ $n k_{n}=n$. 
Evaluating the value of $g^{(n)}$ at 0 gives

$$
g^{(n)}(0)=\sum \frac{n !}{k_{1} ! k_{2} ! \cdots k_{n} !} \varphi^{(k)}\left(\varphi_{z}(0)\right) \prod_{j=1}^{n}\left(\frac{\varphi_{z}^{(j)}(0)}{j !}\right)^{k_{j}} .
$$

Noting that $\varphi_{z}(0)=z$ and $\varphi_{z}^{(j)}(w)=-\left(1-|z|^{2}\right) \bar{z}^{j-1} j !(1-\bar{z} w)^{-j-1}$, we have

$$
\begin{aligned}
g^{(n)}(0) & =\sum \frac{n !}{k_{1} ! k_{2} ! \cdots k_{n} !} \varphi^{(k)}(z) \prod_{j=1}^{n}\left(-\left(1-|z|^{2}\right) \bar{z}^{j-1}\right)^{k_{j}} \\
& =\sum(-1)^{k} \frac{n !}{k_{1} ! k_{2} ! \cdots k_{n} !} \varphi^{(k)}(z)\left(1-|z|^{2}\right)^{k} \bar{z}^{n-k} .
\end{aligned}
$$

The last equality follows from $k_{1}+\cdots+k_{n}=k$ and $k_{1}+2 k_{2}+\cdots+n k_{n}=n$.

Thus

$$
(-1)^{n}\left(1-|z|^{2}\right)^{n} \varphi^{(n)}(z)=g^{(n)}(0)-\sum_{k<n}(-1)^{k} \frac{n !}{k_{1} ! k_{2} ! \cdots k_{n} !} \varphi^{(k)}(z)\left(1-|z|^{2}\right)^{k} \bar{z}^{n-k} .
$$

So

$$
\begin{gathered}
\frac{\left(1-|z|^{2}\right)^{n}\left|\varphi^{(n)}(z)\right|}{1-|\varphi(z)|^{2}} \leq \frac{\left|g^{(n)}(0)\right|}{1-|\varphi(z)|^{2}} \\
+\sum_{k<n} \frac{n !}{k_{1} ! k_{2} ! \cdots k_{n} !} \frac{\left|\varphi^{(k)}(z)\right|\left(1-|z|^{2}\right)^{k}|z|^{n-k}}{1-|\varphi(z)|^{2}} .
\end{gathered}
$$

Let $M_{k}(z)=\frac{\left|\varphi^{(k)}(z)\right|\left(1-|z|^{2}\right)^{k}}{1-|\varphi(z)|^{2}}$. The above inequality gives

$$
M_{n}(z) \leq \frac{\left|g^{(n)}(0)\right|}{1-|\varphi(z)|^{2}}+\sum_{k<n} \frac{n !}{k_{1} ! k_{2} ! \cdots k_{n} !} M_{k}(z) .
$$

We need to estimate $\frac{\left|g^{(n)}(0)\right|}{1-|\varphi(z)|^{2}}$.

Let $\lambda=g(0), h=\varphi_{\lambda} \circ g$. Then $h$ is still an analytic self-map of the unit disk, $h(0)=0$, and $\|h\|_{\infty} \leq 1$. Since $\varphi_{\lambda} \circ \varphi_{\lambda}(z)=z$, we obtain $g=\varphi_{\lambda} \circ h$. The formula of Faa di Bruno again gives

$$
g^{(n)}(w)=\sum \frac{n !}{k_{1} ! k_{2} ! \cdots k_{n} !} \varphi_{\lambda}^{(k)}(h(w)) \prod_{j=1}^{n}\left(\frac{h^{(j)}(w)}{j !}\right)^{k_{j}}
$$

where $k=k_{1}+\cdots+k_{n}$ and the sum is over all $k_{1}, \ldots, k_{n}$ for which $k_{1}+2 k_{2}+\cdots+$ $n k_{n}=n$.

Evaluating $g^{(n)}$ at 0 gives

$$
g^{(n)}(0)=\sum \frac{n !}{k_{1} ! k_{2} ! \cdots k_{n} !} \varphi_{\lambda}^{(k)}(0) \prod_{j=1}^{n}\left(\frac{h^{(j)}(0)}{j !}\right)^{k_{j}}
$$

since $h(0)=0$. Noting $\varphi_{\lambda}^{(k)}(w)=-\left(1-|\lambda|^{2}\right) \bar{\lambda}^{k-1} k !(1-\bar{\lambda} w)^{-k-1}$, the above equality leads to

$$
g^{(n)}(0)=\sum \frac{n !}{k_{1} ! k_{2} ! \cdots k_{n} !}\left[-\left(1-|\lambda|^{2}\right) \bar{\lambda}^{k-1} k !\right] \prod_{j=1}^{n}\left(\frac{h^{(j)}(0)}{j !}\right)^{k_{j}}
$$


Hence

$$
\frac{\left|g^{(n)}(0)\right|}{1-|g(0)|^{2}} \leq \sum \frac{n !}{k_{1} ! k_{2} ! \cdots k_{n} !}|\lambda|^{k-1} k ! \prod_{j=1}^{n}\left(\frac{\left|h^{(j)}(0)\right|}{j !}\right)^{k_{j}} .
$$

Let $a_{n}=\sum_{k<n} \frac{n !}{k_{1} ! k_{2} ! \cdots k_{n} !}$. So far we have shown

$$
M_{n}(z) \leq a_{n} \max _{k<n} M_{k}(z)+\sum \frac{n !}{k_{1} ! k_{2} ! \cdots k_{n} !}|\lambda|^{k-1} k ! \prod_{j=1}^{n}\left(\frac{\left|h^{(j)}(0)\right|}{j !}\right)^{k_{j}} .
$$

Note that $h=\varphi_{\lambda} \circ g, g=\varphi \circ \varphi_{z}$, and $\lambda=g(0)=\varphi(z)$. Then

and

$$
h^{\prime}(w)=\frac{\left(1-|\lambda|^{2}\right) g^{\prime}(w)}{(1-\bar{\lambda} g(w))^{2}}
$$

Let $0<r<1$. Thus

$$
h^{\prime}(w)=\sum_{j=1}^{\infty} \frac{h^{(j)}(0)}{(j-1) !} w^{j-1} .
$$

$$
h^{(j)}(0)=r^{-(j-1)}(j-1) ! \frac{1}{2 \pi} \int_{0}^{2 \pi} h^{\prime}\left(r e^{i \theta}\right) e^{-i(j-1) \theta} d \theta .
$$

So

$$
\begin{aligned}
\left|h^{(j)}(0)\right| & \leq r^{-(j-1)}(j-1) ! \frac{1}{2 \pi} \int_{0}^{2 \pi}\left|h^{\prime}\left(r e^{i \theta}\right)\right| d \theta \\
& \leq r^{-(j-1)}(j-1) ! \frac{1}{2 \pi} \int_{0}^{2 \pi} \frac{\left(1-|\lambda|^{2}\right)\left|g^{\prime}\left(r e^{i \theta}\right)\right|}{\left|1-\bar{\lambda} g\left(r e^{i \theta}\right)\right|^{2}} d \theta \\
& \leq r^{-(j-1)}(j-1) ! \frac{1}{2 \pi} \int_{0}^{2 \pi} \frac{\left(1-\left|\varphi_{\lambda}\left(\left.g\left(r e^{i \theta}\right)\right|^{2}\right)\right| g^{\prime}\left(r e^{i \theta}\right) \mid\right.}{1-\left|g\left(r e^{i \theta}\right)\right|^{2}} d \theta \\
& \leq r^{-(j-1)}\left(1-r^{2}\right)^{-1}(j-1) ! \max _{|w| \leq r} \frac{\left(1-|w|^{2}\right)\left|g^{\prime}(w)\right|}{1-|g(w)|^{2}} \\
& \leq C_{r} \frac{1}{r^{(j-1)}}(j-1) ! \max _{\rho(z, u) \leq r} \frac{\left(1-|u|^{2}\right)\left|\varphi^{\prime}(u)\right|}{1-|\varphi(u)|^{2}}
\end{aligned}
$$

for some constant $C_{r}>0$. The third inequality follows from

$$
\frac{\left(1-|\lambda|^{2}\right)\left(1-\left|g\left(r e^{i \theta}\right)\right|^{2}\right)}{\left|1-\bar{\lambda} g\left(r e^{i \theta}\right)\right|^{2}}=1-\left|\varphi_{\lambda}\left(g\left(r e^{i \theta}\right)\right)\right|^{2} .
$$

The last inequality follows from making the change of variable $u=\varphi_{z}(w)$ and the fact that

$$
\begin{aligned}
& \left(1-|w|^{2}\right)\left|g^{\prime}(w)\right|=\left(1-|w|^{2}\right)\left|\varphi^{\prime}\left(\varphi_{z}(w)\right) \varphi_{z}^{\prime}(w)\right| \\
& \quad=\frac{\left(1-|w|^{2}\right)\left(1-|z|^{2}\right)}{|1-\bar{z} w|^{2}}\left|\varphi^{\prime}\left(\varphi_{z}(w)\right)\right|=\left(1-\left|\varphi_{z}(w)\right|^{2}\right)\left|\varphi^{\prime}\left(\varphi_{z}(w)\right)\right| .
\end{aligned}
$$

Hence

$$
\frac{\left|h^{(j)}(0)\right|}{j !} \leq\left[j r^{(j-1)}\left(1-r^{2}\right)\right]^{-1} \max _{\rho(z, u) \leq r} \frac{\left(1-|u|^{2}\right)\left|\varphi^{\prime}(u)\right|}{1-|\varphi(u)|^{2}} .
$$

The Schwarz-Pick estimate gives

$$
\frac{\left(1-|u|^{2}\right)\left|\varphi^{\prime}(u)\right|}{1-|\varphi(u)|^{2}} \leq 1
$$


for each $u \in D$. Thus

$$
\begin{aligned}
& \sum \frac{n !}{k_{1} ! k_{2} ! \cdots k_{n} !}|\lambda|^{k-1} k ! \prod_{j=1}^{n}\left(\frac{\left|h^{(j)}(0)\right|}{j !}\right)^{k_{j}} \\
& \quad \leq \sum \frac{n !}{k_{1} ! k_{2} ! \cdots k_{n} !}|\lambda|^{k-1} k ! r^{k-n}\left(1-r^{2}\right)^{-k} \max _{\rho(z, u) \leq r} \frac{\left(1-|u|^{2}\right)\left|\varphi^{\prime}(u)\right|}{1-|\varphi(u)|^{2}} .
\end{aligned}
$$

Let $b_{n, r}=\sum \frac{n !}{k_{1} ! k_{2} ! \cdots k_{n} !} k ! r^{k-n}\left(1-r^{2}\right)^{-k}$. The above inequality gives

$$
M_{n}(z) \leq a_{n} \max _{k<n} M_{k}(z)+b_{n, r} \max _{\rho(z, u) \leq r} \frac{\left(1-|u|^{2}\right)\left|\varphi^{\prime}(u)\right|}{1-|\varphi(u)|^{2}} .
$$

By the induction, we conclude that

$$
M_{n}(z) \leq M_{n, r} \max _{\rho(z, u) \leq r} \frac{\left(1-|u|^{2}\right)\left|\varphi^{\prime}(u)\right|}{1-|\varphi(u)|^{2}}
$$

to complete the proof.

\section{Hyperbolic DeRIVATIVES}

In this section we will first show that the hyperbolic derivative of an analytic self-map $\varphi$ of the unit disk equals $\left|\tau_{\varphi}(z)\right|$. Then we will show that $\tau_{\varphi}(z)$ is Lipschitz with respect to the pseudo-hyperbolic metric.

Theorem 5. Let $\varphi: D \rightarrow D$ be an analytic self-map. Then, for each point $z \in D$, the hyperbolic derivative of $\varphi$ is equal to

$$
\lim _{\beta(z, w) \rightarrow 0} \frac{\beta(\varphi(z), \varphi(w))}{\beta(z, w)}=\left|\tau_{\varphi}(z)\right| .
$$

Proof. Assume that $\varphi$ is not constant. For each fixed $z \in D, \rho(\varphi(z), \varphi(w))$ converges to zero as $\beta(w, z)$ converges to zero because $\varphi$ is continuous in $D$ and $|\varphi(z)|<1$. Thus

$$
\lim _{\beta(z, w) \rightarrow 0} \frac{\beta(\varphi(z), \varphi(w))}{\beta(z, w)}=\lim _{\beta(z, w) \rightarrow 0} \frac{\beta(\varphi(z), \varphi(w))}{\rho(\varphi(z), \varphi(w))} \frac{\rho(\varphi(z), \varphi(w))}{\rho(z, w)} \frac{\rho(z, w)}{\beta(z, w)} .
$$

Both the first and third factors of the product on the right converge to one. Now the second factor is

$$
\frac{\rho(\varphi(z), \varphi(w))}{\rho(z, w)}=\frac{|\varphi(z)-\varphi(w)|}{|z-w|} \frac{|1-\bar{z} w|}{|1-\overline{\varphi(z)} \varphi(w)|} .
$$

Thus

$$
\lim _{\beta(z, w) \rightarrow 0} \frac{\rho(\varphi(z), \varphi(w))}{\rho(z, w)}=\frac{\left|\varphi^{\prime}(z)\right|\left(1-|z|^{2}\right)}{1-|\varphi(z)|^{2}} .
$$

So

$$
\lim _{\beta(z, w) \rightarrow 0} \frac{\beta(\varphi(z), \varphi(w))}{\beta(z, w)}=\frac{\left|\varphi^{\prime}(z)\right|\left(1-|z|^{2}\right)}{1-|\varphi(z)|^{2}} .
$$

This completes the proof.

For each $n$, define

$$
\tau_{\varphi, n}(z)=\frac{\left(1-|z|^{2}\right)^{n} \varphi^{(n)}(z)}{1-|\varphi(z)|^{2}} .
$$


Theorem 6. Let $\varphi$ be an analytic self-map of the unit disk $D$. Then for each $n$, $\tau_{\varphi, n}(z)$ is Lipschitz with respect to the pseudohyperbolic metric. More precisely,

$$
\left|\tau_{\varphi, n}(z)-\tau_{\varphi, n}(w)\right| \leq C_{n} \rho(z, w)
$$

for any $z, w \in D$. Here $C_{n}$ is a positive constant only depending on $n$.

Proof. Suppose that $f$ is a differentiable function on the unit disk. Let $\partial_{z} f=\frac{\partial f}{\partial z}$ and $\partial_{\bar{z}} f=\frac{\partial f}{\partial \bar{z}}$. Note that $\tau_{\varphi, n}(z)$ is differentiable on the unit disk. Easy calculations give

$$
\partial_{\bar{z}} \tau_{\varphi, n}(z)=\frac{-z n\left(1-|z|^{2}\right)^{n-1} \varphi^{(n)}(z)\left(1-|\varphi(z)|^{2}\right)+\left(1-|z|^{2}\right)^{(n)} \varphi^{(n)}(z) \overline{\varphi^{\prime}(z)} \varphi(z)}{\left(1-|\varphi(z)|^{2}\right)^{2}}
$$

and

$$
\begin{aligned}
\partial_{z} \tau_{\varphi, n}(z)= & \frac{1}{\left(1-|\varphi(z)|^{2}\right)^{2}}\left\{\left[\left(1-|z|^{2}\right)^{n} \varphi^{(n+1)}(z)-\bar{z} n\left(1-|z|^{2}\right)^{n-1} \varphi^{(n)}(z)\right]\right. \\
& \left.\times\left(1-|\varphi(z)|^{2}\right)+\left(1-|z|^{2}\right)^{n} \varphi^{(n)}(z) \varphi^{\prime}(z) \overline{\varphi(z)}\right\} .
\end{aligned}
$$

Thus

$$
\begin{aligned}
& \left|\partial_{\bar{z}} \tau_{\varphi, n}(z)\right| \leq \frac{1}{1-|z|^{2}}\left[\frac{n\left(1-|z|^{2}\right)^{n}\left|\varphi^{(n)}(z)\right|}{1-|\varphi(z)|^{2}}\right. \\
& \quad+|\varphi(z)|\left[\frac{\left(1-|z|^{2}\right)\left|\varphi^{\prime}(z)\right|}{1-|\varphi(z)|^{2}}\right]\left[\frac{\left(1-|z|^{2}\right)^{n}\left|\varphi^{(n)}(z)\right|}{1-|\varphi(z)|^{2}}\right] \leq \frac{(n+1) M_{n}}{1-|z|^{2}},
\end{aligned}
$$

where the last inequality follows from Theorem 3 , and

$$
\begin{aligned}
& \left|\partial_{z} \tau_{\varphi, n}(z)\right| \leq \frac{1}{1-|z|^{2}}\left\{\frac{\left(1-|z|^{2}\right)^{n+1}\left|\varphi^{(n+1)}(z)\right|}{1-|\varphi(z)|^{2}}+n \frac{\left(1-|z|^{2}\right)^{n}\left|\varphi^{n}(z)\right|}{1-|\varphi(z)|^{2}}\right. \\
& \left.+|\varphi(z)|\left[\frac{\left(1-|z|^{2}\right)^{n}\left|\varphi^{(n)}(z)\right|}{1-|\varphi(z)|^{2}}\right]\left[\frac{\left(1-|z|^{2}\right)\left|\varphi^{\prime}(z)\right|}{1-|\varphi(z)|^{2}}\right]\right\} \leq \frac{M_{n+1}+(n+1) M_{n}}{1-|z|^{2}}
\end{aligned}
$$

where the last inequality follows from Theorem 3 . Given $z$ and $w$ in $D$, let $\gamma(t)$ : $[0,1] \rightarrow D$ be a smooth curve to connect $z$ and $w$, i.e.,

$$
\begin{aligned}
\left|\tau_{\varphi, n}(z)-\tau_{\varphi, n}(w)\right|= & \left|\int_{0}^{1} \frac{d \tau_{\varphi, n}(\gamma(t))}{d t} d t\right| \\
& \leq \int_{0}^{1}\left|\frac{d}{d t} \tau_{\varphi, n}(\gamma(t))\right| d t \\
& \leq \int_{0}^{1}\left[\left|\partial_{z} \tau_{\varphi, n}(\gamma(t))\right|\left|\frac{d \gamma(t)}{d t}\right|+\left|\partial_{\bar{z}} \tau_{\varphi, n}(\gamma(t))\right|\left|\frac{d \gamma(t)}{d t}\right|\right] d t
\end{aligned}
$$

where the last inequality follows from the first chain rule:

$$
\frac{d}{d t} \tau_{\varphi, n}(\gamma(t))=\partial_{z} \tau_{\varphi}(\gamma(t)) \frac{d \gamma(t)}{d t}+\partial_{\bar{z}} \tau_{\varphi, n}(\gamma(t)) \frac{\overline{d \gamma(t)}}{d t} .
$$

Combining the above estimates gives

$$
\left|\tau_{\varphi, n}(z)-\tau_{\varphi, n}(w)\right| \leq \int_{\gamma} \frac{M_{n+1}+2(n+1) M_{n}}{1-|\gamma(t)|^{2}} d|\gamma(t)| .
$$


If we choose $\gamma$ to be a geodesic to connect $z$ and $w$, then the above inequality gives

$$
\begin{aligned}
\mid \tau_{\varphi, n}(z) & -\tau_{\varphi, n}(w) \mid \leq\left(M_{n+1}+2(n+1) M_{n}\right) \beta(z, w) \\
& \leq \frac{\left(M_{n+1}+2(n+1) M_{n}\right) \rho(z, w)}{1-\rho(z, w)^{2}}
\end{aligned}
$$

The last inequality comes from the fact that for all $0<x<1$,

$$
\frac{1}{2} \ln \frac{1+x}{1-x} \leq \frac{x}{1-x^{2}} .
$$

If $|\rho(z, w)|<1 / 8$, the above inequality gives

$$
\left|\tau_{\varphi, n}(z)-\tau_{\varphi, n}(w)\right| \leq 2\left(M_{n+1}+2(n+1) M_{n}\right) \rho(z, w) .
$$

If $|\rho(z, w)| \geq 1 / 8$, we have $8|\rho(z, w)| \geq 1$, and

$$
\left|\tau_{\varphi, n}(z)-\tau_{\varphi, n}(w)\right| \leq \max \left\{\left|\tau_{\varphi, n}(z)\right|,\left|\tau_{\varphi, n}(w)\right|\right\} \leq M_{n} \leq 8 M_{n} \rho(z, w) .
$$

Choosing $C_{n}=\max \left\{2\left(M_{n+1}+2(n+1) M_{n}\right), 8 M_{n}\right\}$, we have

$$
\left|\tau_{\varphi, n}(z)-\tau_{\varphi, n}(w)\right| \leq C_{n} \rho(z, w)
$$

to complete the proof.

Theorem 6 has an application to closed-range composition operators on the Bloch space.

Hoffman [6] showed that $\left(1-|z|^{2}\right)^{n} \varphi^{(n)}(z)$ continuously extends to the maximal ideal space of $H^{\infty}$. Let $\mathcal{G}$ be the subset of the maximal ideal space of $H^{\infty}$ consisting of nontrivial Gleason parts. As a corollary of a result in [1] and Theorem 1.2 [4, we have the following result.

Corollary 1. Suppose that $\varphi$ is an analytic self-mapping of the unit disk. Then $\tau_{\varphi, n}(z)$ admits a continuous extension to $\mathcal{G}$.

\section{ADDENDUM}

After we finished this paper, we obtained K. Stroethoff's paper [14, which showed that

$$
\rho\left(\left|\tau_{\varphi}(z)\right|,\left|\tau_{\varphi}(w)\right|\right) \leq 2 \rho(z, w)
$$

for $z, w \in D$. This generalizes Beardon's result [3]: If $\varphi(0)=0$, then

$$
\rho\left(\tau_{\varphi}(0), \tau_{\varphi}(w)\right)<2 \rho(0, w)
$$

for $w \in D$. We thank K. Stroethoff.

\section{REFERENCES}

[1] S. Axler and K. Zhu, Boundary behavior of derivatives of analytic functions, Michigan Math. J. 39 (1992), 129-143. MR 93e:30073

[2] L. V. Ahlfors, Conformal invariants: topics in geometric function theory, McGraw-Hill, New York, 1973. MR 50:10211

[3] A. Beardon, The Schwarz-Pick Lemma for derivatives, Proc. Amer. Math. Soc. 125 (1997), 3255-3256. MR 97m:30062

[4] A. Brudnyi, Topology of the maximal ideal space of $H^{\infty}$, J. Funct. Analysis 189 (2002), 21-52. MR 2003c: 46066

[5] J. Garnett Bounded analytic functions, Academic Press, New York, 1981. MR 83g:30037

[6] K. Hoffman, Bounded analytic functions and Gleason parts, Ann. Math. 86 (1967), 74-111. MR 35:5945

[7] P. Ghatage, J. Yan and D. Zheng, Composition operators with closed range on the Bloch space, Proc. Amer. Math. Soc. 129 (2001), 2039-2044. MR 2002a:47034 
[8] B. MacCluer, K. Stroethoff, and R. Zhao, Generalized Schwarz-Pick estimates, Proc. Amer. Math. Soc. 131 (2003), 593-599. MR 2003g:30038

[9] K. Madigan and A. Matheson, Compact composition operators on the Bloch space, Trans. Amer. Math. Soc. 347 (1995), 2679-2687. MR 95i:47061

[10] A. Montes-Rodríguez, The essential norm of a composition operator on Bloch spaces, Pacific J. Math. 188 (1999), 339-351. MR 2000d:47044

[11] S. Ohno, K. Stroethoff and R. Zhao, Weighted composition operators between Bloch-type space, Rocky Mountain J. Math. 33 (2003), 191-215. MR 2004d:47058

[12] Ch. Pommerenke, Boundary behaviour of conformal maps, Springer-Verlag 299, New York, 1991. MR 95b:30008

[13] S. Roman, The Formula of Faa di Bruno, Amer. Math. Monthly 87 (1980), 805-809. MR 82d:26003

[14] K. Stroethoff, Lecture notes on The Schwarz-Pick Lemma for derivatives, Preprint.

Department of Mathematics, Cleveland State University, Cleveland, Ohio 44115

E-mail address: p.ghatage@csuohio.edu

Department of Mathematics, Vanderbilt University, Nashville, Tennessee 37240

E-mail address: zheng@math.vanderbilt.edu 